\title{
PERFIL EPIDEMIOLÓGICO DE CRIANCAS NASCIDAS DE MÃES HIV POSITIVAS EM REGIÃO METROPÓLITANA DA AMAZÔNIA
}

\section{Núria Jussara Lima Silva}

Fisioterapeuta. Graduada pela Universidade da Amazônia (UNAMA), Belém (PA), Brasil.

\section{Flavia Maciel Lobato}

Fisioterapeuta. Graduada pela Universidade da Amazônia (UNAMA), Belém (PA), Brasil.

\section{Nayan Leonardo Sousa Lopes}

Fisioterapeuta. Graduada pela Universidade da Amazônia (UNAMA), Belém (PA), Brasil.

\section{Ana Júlia Cunha Brito}

Fisioterapeuta. Mestre em Desenvolvimento e Meio Ambiente Urbano pela Universidade da Amazônia, Docente da Faculdade Cosmopolita, Belém (PA), Brasil.

\section{George Alberto da Silva Dias}

Fisioterapeuta, Doutor em Doenças Tropicais pelo NMT/UFPA/PA, Docente do Departamento de Ciências do Movimento Humano da Universidade do Estado do Pará, Belém (PA), Brasil.

\section{Biatriz Araújo Cardoso}

Fisioterapeuta, Doutora em Ciências pelo Programa de Medicina Tropical pelo Instituto Oswaldo Cruz/ FIOCRUZ, Docente do Departamento de Ciências do Movimento Humano da Universidade do Estado do Pará, Belém (PA), Brasil.

\section{Dayse Danielle Oliveira Silva}

Fisioterapeuta. Doutora em Ciências da Reabilitação pela Universidade Nove de Julho, Docente do Departamento de Ciências do Movimento Humano da Universidade do Estado do Pará, Belém (PA), Brasil.
RESUMO: O objetivo desta pesquisa é descrever o perfil epidemiológico de crianças nascidas de mães HIV positivas, atendidas na Unidade de Referência Materno-Infantil e do Adolescente (UREMIA), em Belém-Pará no ano de 2015. Estudo descritivo transversal, com análise dos fatores que descrevem o cenário de nascimento de 125 crianças de mães HIV positivas. Utilizaram-se os testes $G$ (Aderência) e Qui-quadrado para a análise estatística $(p \leq 0,05)$. Do total de $125,96 \%$ das mães realizaram pré-natal com duas a seis consultas e grande parte descobriu o HIV durante a gestação (39\%) ou no parto (14\%), e, $75 \%$ fizeram o uso de antirretrovirais (AZT) após o diagnóstico da infecção. Apenas 6\% das crianças possuem HIV, $6 \%$ não usaram AZT e 5\% amamentaram. Assim, as mães deste estudo são da zona urbana, realizaram o pré-natal e fizeram uso precoce do AZT, quanto aos lactentes nasceram de parto normal, fizeram uso do AZT podendo contribuir para baixa transmissão vertical.

PALAVRAS-CHAVE: Sorodiagnóstico da AIDS; Transmissão vertical de doença infecciosa; antirretrovirais.

\section{EPIDEMIOLOGIC PROFILE OF CHILDREN BORN OF HIV-POSITIVE MOTHERS IN A METROPOLITAN REGION IN THE AMAZON REGION}

ABSTRACT: Current research describes the epidemiologic profile of children born of HIV positive mothers attended to at the Mother-Infant and Adolescent Reference Unit (UREMIA) in Belém PA Brazil, in 2015. The transversal description analyzed factors that describe the birth scenario of 125 children born of HIV positive mothers. G (Adherence) and Chi-Square tests were employed for statistical analysis $(\mathrm{p} \leq 0.05)$. Further, $96 \%$ of the 125 mothers underwent the pre-natal exam, with two to six visits. Many (39\%) discovered they were infected by HIV during pregnancy or in childbirth (14\%), whilst $75 \%$ used anti-retroviral agents (AZT) for the diagnosis of the infection. Only $6 \%$ of the children had HIV; 6\% did not use AZT and 5\% breastfed their children. The mothers in current study belonged to the urban zone, undertook pre-natal tests and early used AZT. Since breastfed children were born by normal birth and the mother used AZT, the two factors may have contributed towards low vertical transmission.

KEY WORDS: AIDS Serodiagnosis; Infectious Disease Transmission, Vertical; Anti-Retroviral Agents. 


\section{INTRODUÇão}

A Síndrome da Imunodeficiência Adquirida (AIDS) é uma das maiores preocupações de saúde do mundo. Apesar das evoluções significativas na prevenção de novas infecções pelo vírus da imunodeficiência humana (HIV) e na redução de mortes relacionadas à doença, ainda é crescente a quantidade de pessoas vivendo com o vírus ${ }^{1}$.

No Brasil, a taxa do HIV na região Norte tende a crescer, em contraste com a região Sudeste que tende a diminuir. Em Belém, capital do Estado do Pará, o aumento da incidência da doença foi de 14,3 para 53,5 casos/100 mil habitantes entre 1997 e 2009, refletindo um problema alarmante e desafiador na saúde pública pelos fatores socioambientais, culturais e econômicos dessa região que são fatores propícios para a aquisição desta infecção vi$\mathrm{ral}^{2}$.

Quando se relata infecção pelo vírus em mulheres grávidas, o problema se torna ainda maior. Segundo Preussler e Eidt ${ }^{3}$, muitas delas só descobrem a infecção durante a gestação ou engravidam já sabendo da contaminação pelo vírus, o que aumenta os riscos para a transmissão vertical (TV). Esta ocorre quando o agente infeccioso é transmitido da mãe para o filho no período gestacional, no trabalho de parto, durante o parto ou pelo leite materno. $\mathrm{O}$ número de mulheres em idade reprodutiva infectadas pelo HIV é agravante, e no intuito de diminuir o número de crianças portadoras da doença, em 1996 foi implantado o Programa Nacional em HIV/AIDS, no qual assegura o teste para detecção viral como exame de rotina no pré-natal.

No período de 2000 a 2009, o número de gestantes HIV positivas, na faixa etária de 20 a 29 anos, atingiu cerca de 47.705 mulheres. Esse número evidencia a probabilidade de aumento TV, no qual o Estado do Pará é responsável por 60 a 86\% dos casos na região Norte do Brasil ${ }^{4}$.

A transmissão vertical é a principal via de infecção pelo HIV em crianças menores de 13 anos no mundo. Sendo que cerca de $35 \%$ ocorre durante a gestação, $65 \%$ no periparto e de 7 a $22 \%$ no período de aleitamento materno 5 .

Sherlock et $a^{6}$ destacam que além do teste so- rológico para HIV no pré-natal, outras medidas são empregadas ao se detectar a infecção viral, como o uso de drogas antirretrovirais na gestação, o aconselhamento do parto cesáreo, o uso de Iquego-Zidovudina (AZT), o uso de medicação apropriada ao recém-nascido (RN) e recomendação da inibição do aleitamento materno, substituindo-o por leite artificial. Vale ressaltar a importância do acompanhamento do RN exposto ao HIV, por equipe multiprofissional, a fim de garantir a integralidade dos serviços disponíveis por meio do encaminhamento à consulta após alta hospitalar da maternidade, para observar o desenvolvimento viral durante os dois primeiros anos de vida através de exames clínicos e laboratoriais.

A manifestação clínica do HIV se diferencia entre adultos e crianças no curso da sua infecção, visto que a ação viral no sistema nervoso em desenvolvimento pode acometer qualquer parte do neuroeixo da criança, podendo levar a uma encefalopatia. Já em comum apresentam alterações da função imunológica, que favorecem maior risco de infecções secundárias, onde as crianças infectadas por via vertical sofrem progressão acelerada e severa da doença, principalmente nos três primeiros anos de vida, com evolução de oito a dez anos. Contudo, um grupo de aproximadamente $20 \%$ morre antes de completar dois anos de idade, com sinais de imunodeficiência grave e/ou encefalopatia associada?.

Diante disso, este estudo objetivou descrever o perfil epidemiológico de crianças nascidas de mães HIV positivas, atendidas na Unidade de Referência Materno-Infantil e do Adolescente (UREMIA), em Belém-Pará no ano de 2015. Uma vez que o levantamento dos vários fatores influenciadores da transmissão vertical favorecem a interpretação do aumento no número de crianças com o vírus ${ }^{8}$.

\section{METODOLOGIA}

\section{PRINCÍPIOS ÉTICOS}

A pesquisa teve início após aprovação do Comitê de Ética em Pesquisa da Universidade da Amazônia (Unama) (parecer $n^{0}$ 1.286.792). Trata-se de um estudo analítico do tipo transversal, seguindo as normas da re- 
solução 466/12 do Conselho Nacional de Saúde, relativa à pesquisa com seres humanos. A amostra foi composta por crianças nascidas de mães HIV positivas que compareceram à Unidade de Referência Materno Infantil e do Adolescente (Uremia), no Estado do Pará, sendo selecionados por meio da amostragem não probabilística por conveniência. Todos os participantes assinaram o consentimento formal para participar do estudo.

\section{AMOSTRA}

Participaram do estudo 125 crianças com coleta das informações no período de janeiro a abril de 2016. Foram incluídos no estudo mães HIV positivas e seus respectivos filhos, de 6-18 meses, ambos os sexos, submetidos ao tratamento com antirretroviral e matriculados na Uremia. E excluídas aquelas que possuíam doenças neuromotoras, cardiorrespiratórias e que não tivessem utilizado terapia antirretroviral.

\section{PROCEDIMENTOS}

Inicialmente, as informações foram colhidas da ficha demográfica de acompanhamento na Uremia, no Programa Pediatria-Doenças Infecto Parasitárias (DIP), com posterior entrevista com as mães destas crianças. Colheram-se informações demográficas e epidemiológicas referentes ao sexo e à idade das crianças e dos pais, endereço, escolaridade, ocupação, história do pré-natal, do parto, características do recém-nascido, antecedentes familiares, bem como informações sobre o tipo de transmissão do HIV em relação à mãe (transmissão sexual, múltiplos parceiros ou outras exposições), ao parceiro (transmissão sexual, múltiplos parceiros, bissexual ou outras exposições), e em relação à criança (TV, amamentação cruzada, abuso sexual, transfusão ou acidental). Além disso, levantaram-se informações sobre o pré-natal, identificando o local, o número de consultas, o número de gestações, partos, abortos e a data do diagnóstico de infecção por HIV (antes da gravidez, durante a gravidez, no parto - teste rápido, após o parto ou desconhecido). Quanto ao uso de antirretroviral (AZT) coletaram-se informações associadas à gestação (o início do uso e o mês de gestação), ao parto e às complicações obstétricas.
Referente ao parto foram coletadas informações sobre o local, o tipo de parto (parto normal ou cesárea) e a idade gestacional (a termo, pré-termo, pós- termo). E por fim, dados referentes às características do recém-nascido, buscaram-se informações sobre o teste para identificação de pacientes com HIV, complicações no período neonatal e uso de antirretroviral no período neonatal.

\section{ANÁLISE DOS DADOS}

Adotou-se o software Excel ${ }^{\circledR} 2010$ para entrada dos dados e confecção das tabelas, bem como o BioEstat 5.0 na análise estatística. As variáveis categóricas foram apresentadas como frequências e as numéricas por meio de medidas de tendência central (média) e dispersão (desvio-padrão). Para verificar a normalidade dos dados, utilizou-se o teste D'Agostino-Pearson. Para as variáveis não paramétricas foram usados os testes $\mathrm{G}$ (Aderência) $\mathrm{e}$ o Qui-quadrado, adotando o nível alfa de significância de $5 \%(\mathrm{p} \leq 0,05)$.

\section{RESULTADOS}

Das 125 crianças nascidas de mães HIV positivas, 65,6\% (82/125) residem na área urbana, 50,4\% (63/125) das mães encontravam-se na faixa etária de 18 a 26 anos, correspondendo à média de idade de 27,2 anos, 52,8\% (66/125) possuem o ensino médio. Com relação aos pais, $46,4 \%$ (58/125) estão na faixa de 28 anos ou mais, com média de idade de 30,9 anos, 32,0\% (40/125) também possuem ensino médio (Tabela 1).

Com relação à exposição sexual para o HIV, $70,4 \%$ (88/125) das mães tiveram de um a oito parceiros, enquanto que $20 \%(25 / 125)$ dos pais tiveram mais de dez parceiras. Além disso, 92,4\% (61/66) das mães possuem tatuagem ou piercing e 7,6\% (5/66) já utilizaram drogas inalatórias, para os pais $66,0 \%(31 / 47)$ possuem tatuagens ou piercing, 34,0\% (16/47) usaram drogas inalatórias e $5,0 \%$ (6/125) são bissexuais. Quanto à positividade da infecção para o HIV, 39,2\% (49/125) são negativos e 24,8\% (31/125) são soropositivos. Com relação à exposição das crianças na aquisição do HIV 100\% foi pela transmissão vertical (Tabela 2).

Quanto aos dados relacionados à história do pré- 
-natal, observou-se que $96,0 \%$ (120/125) das mães realizaram o pré-natal, sendo 55,2\% (69/125) na Uremia e 48,8\% (61/125) realizaram de duas a seis consultas médicas. Em relação à descoberta da infecção para o HIV, 44,0\% (55/125) delas descobriram antes da gravidez, 75,2\% (94/125) fizeram o uso do AZT e 36,8\% (46/125) entre 0 a cinco meses de gestação, sendo que 81,6\% (102/125) obtiveram o acesso ao AZT injetável (Tabela 3).

Os partos foram realizados em diferentes hospitais do Estado do Pará, assim, nota-se que 72,0\% (90/125) ocorreram em hospitais públicos, 12,8\% (16/125) realizaram parto normal, e, apesar de 80,8\% (101/125) das crianças nascerem dentro da idade gestacional considerada normal (a termo), 5,6\% (7/125) foram consideradas pré-termo (Tabela 4).

Na Tabela 5 constam os dados referentes às características do recém-nascido, onde 5,6\% (7/125) possuem HIV. Ao nascer apenas 4,0\% (5/125) não usou AZT xarope e 4,8\% (6/125) foram amamentadas. Quanto aos antecedentes familiares $37,7 \%(23 / 61)$ possuem familiares com AIDS e 45,9\% (28/61) com tuberculose.

\section{DISCUSSÃO}

Neste estudo, das 125 crianças nascidas de mães HIV positivas, 65,6\% residiam na área urbana e eram filhas de mães com média de idade de 27,2 anos, cujos principais fatores de risco para infecção pelo HIV consistia em exposição sexual a múltiplos parceiros $(70,4 \%) \mathrm{e}$ presença de tatuagens e/ou pearcing (92,4\%), e, 52,8\% possuíam apenas o ensino médio. Segundo Fonseca et al. ${ }^{9}$ a transmissão vertical do HIV está diretamente relacionada a alguns fatores como a baixa escolaridade das gestantes, o baixo número de pré-natal e dificuldades na realização do teste rápido no pré-parto. Esses dados referentes à região Norte do Brasil mostram que a diretriz preconizado pelo Ministério da Saúde não é atingida, o que pode influenciar diretamente à persistência dos altos índices da TV, tornando-se um sério risco de saúde pública.

Um estudo realizado no município de Gurupi ${ }^{10}$, também localizado na região Norte, alcançou números semelhantes ao presente estudo, no qual a idade média das gestantes com HIV foi 25,47 anos. Outra pesquisa realizada na África subsaariana ${ }^{11}$ obteve, como idade média das gestantes, 28,3 anos e com nível de escolaridade baixa. Estes dados corroboram com o estudo ${ }^{12}$, no qual verificaram que a prevalência do HIV nas mulheres grávidas pode estar relacionada com os aspetos comportamentais dos seus parceiros em relação ao uso de preservativos, a desigualdade de gênero, a violência sexual, o casamento precoce e o acesso limitado à educação.

Das mães das crianças incluídas nesta pesquisa, 96,0\% realizaram o pré-natal. Embora a adesão ao pré-natal pela população pesquisada tenha se mostrado elevada, o quantitativo de consultas durante a gestação não está de acordo com o número mínimo de atendimentos estabelecido pelo Ministério da Saúde, essas informações corroboram com o estudo realizado no Rio de Janeiro ${ }^{13}$, no qual o número de consultas do pré-natal de gestantes com infecção pelo HIV foi inferior a seis consultas e 63,5\% tiveram seus filhos de parto normal aumentando as chances de TV. Lemos et al. ${ }^{14}$ observaram que ao aumentar o número de consultas no pré-natal, diminuem os riscos de TV, contudo existem outros protocolos que também devem ser cumpridos como o uso do AZT.

Vale ressalta que $44,0 \%$ das mães descobriram a infecção pelo HIV antes da gravidez e 75,2\% fizeram o uso do AZT, porém 4,0\% das crianças não realizaram o AZT xarope até 42 dias de vida. Na Nigéria, os autores ${ }^{15}$ analisaram algumas barreiras relacionadas ao não oferecimento do AZT nas primeiras semanas de vida do recém-nascido, sendo um dos motivos, a falta de comunicação com os profissionais de saúde, o constrangimento e o esquecimento, o que segundo Andreatta e Sammarco ${ }^{16}$, contribuem para um prognóstico desfavorável, podendo gerar alterações no desenvolvimento infantil.

Esse estudo destaca que $75 \%$ das mães receberam o AZT, $51,5 \%$ durante a gravidez, $6,4 \%$ no parto e $22,4 \%$ já faziam uso do medicamento antes da gravidez, sendo que a maioria dessas só descobriu a infecção pelo vírus em outro período gestacional, esses dados expõem o risco de TV, pois indicam que a maioria das mulheres entrevistadas só teve conhecimento da contaminação durante o pré-natal. Uma pesquisa realizada em Camarões ${ }^{17}$ analisou a taxa de sobrevivência das crianças nascidas de mães HIV positivas, e constatou que as mães que recebe- 
ram o AZT no primeiro trimestre da gravidez aumentaram as chances de sobrevida das crianças em cerca de $84,3 \%$, em oposição, as que não receberam 43,3\%.

As informações apresentadas pela pesquisa avultam sobre as falhas nos serviços de saúde quanto aos cumprimentos das medidas de prevenção da TV, pois 13,0\% das crianças nasceram de parto normal, 4,0\% não receberam o AZT ao nascer, 5,6\% nasceram prematuros e 5,0\% foram amamentados, revelando a necessidade de uma atenção especial direcionada à assistência no pré-natal e orientações após o parto. Contraponto o estudo ${ }^{18}$ realizado em um hospital público do Estado do Pará, onde dos 70 recém-nascidos, filhos de mães HIV positivas, $4,0 \%$ nasceram de parto normal e todos receberam AZT por via oral durante seis semanas após o nascimento, $11 \%$ nasceram prematuros e nenhum lactente foi amamentado, por se tratar de um hospital de referência no Estado, sobressai a importância do diagnóstico precoce e o atendimento especializado para essas mães a fim de minimizar os riscos da TV.

Embora se tenha obtido resultados relevantes em relação à necessidade de ações contínuas e específicas na atenção à saúde materno-infantil e relação com a infecção pelo HIV, é notório que este estudo apresente algumas limitações, estando sobretudo relacionadas ao viés de informações quanto aos preconceitos e medos que rondam o assunto, pois se trata de uma infecção ainda estigmatizada na sociedade e o preenchimento incompleto dos prontuários dos pacientes, podendo ocultar dados mais alarmantes da real situação da saúde materno-infantil.

\section{CONCLUSÃO}

O cenário observado se assemelha aos descritos nas demais regiões do Brasil, onde as mães são residentes da zona urbana, realizaram o pré-natal e tiveram relações com parceiros bissexuais. Grande parte das gestantes fez uso do AZT ainda na gestação e os lactentes nos primeiros meses de vida, o que provavelmente, associado ao parto cesáreo, contribuiu para a baixa transmissão vertical.

Os dados encontrados podem subsidiar o desenvolvimento de estratégias preventivas voltadas para saúde da mulher e da criança, e, também, fortalecer as já preconizadas pelo Ministério da Saúde, visto que ainda há crianças sem acesso ao AZT após o nascimento, assim como orientar às mães quanto à contraindicação da amamentação, diminuindo as taxas de prevalência da transmissão vertical.

\section{REFERÊNCIAS}

1. Lima ACMACC, Costa CC, Teles LMR, Damasceno AKC, Oriá MOB. Avaliação epidemiológica da prevenção da transmissão vertical do HIV. Acta. Paul. Enferm. 2014;27(4):311-8.

2. Abati PAM, Segurado AC. Testagem Anti-HIV e Estádio Clínico na Admissão de Indivíduos em Serviço de Saúde Especializado, Pará, Brasil. Revista Saúde Pública. 2015;49(16):1-8.

3. Preussler GMI, Eidt OR., Vivenciando as Adversidades do Binômio Gestação e HIV/AIDS. Revista Gaúcha de Enfermagem. 2007;28(1):117-125.

4. Menezes LSH, Palácios VRCM, Alcântara MSV, Bichara CN. Prevalência da Infecção por HIV em grávidas no Norte do Brasil. DST - J bras Doenças Sex Transm. 2012;24(4):250-254.

5. Pereira DA, Horiy DM, Proença EO, Santos ASA. Expectativas de mães soropositivas em processo de pesquisa de transmissão vertical do HIV, em Sorocaba/ SP. Rev. Fac. Ciênc. Méd. Sorocaba. 2014;16(1):11-14.

6. Sherlock MSM, Cardoso MVLML, Lopes MMCO, Lélis ALPA, Oliveira NR. Imunização em criança exposta ou infectada pelo HIV em um serviço de imunobiológicos especiais. Esc. Anna Nery. 2011;15(3):573-580.

7. Andreatta LB, Sammarco GN. Aspecto clínico de crianças portadoras da síndrome da imunodeficiência adquirida por transmissão vertical: uma revisão bibliográfica. Revista Saúde e Pesquisa. 2012;5(2):418-423.

8. Miranda AE, Pereira GFM, Araujo MAL, Silveira MF, Tavares LL, Silva LCF, et al. Avaliação da cascata de cuidado na prevenção da transmissão vertical do HIV no Brasil. Cad. Saúde Pública. 2016;32(9):e00118215.

9. Fonseca FF, Jesus BR, Rocha KT, Silva SSS, Andrade DCS, Costa FM. A transmissão vertical do HIV no Brasil: uma revisão de literatura. Revista Eletrônica Gestão \& Saúde. 2015;1:533-48. 
10. Tavares R, Gontijo EEL, Santos ES, Jubé JKB, Mazutti AR, Silva MG. Perfil de grávidas com sífilis, HIV+ ou hepatite B em Gurupi, Tocantins. Revista Saúde e Pesquisa. 2014;7(1):35-45.

11. Linguissi LSG, Bisseye C, Sagna T, Nagalo BM, Ouermi D, Djigma FW, Pignatelli S, Sia JD, Pietra V, Moret R, Nikiema JB, Simpore J. Efficiency of HAART in the prevention of mother to children HIV-1 transmission at Saint Camille medical centre in Burkina Faso, West Africa. Asian Pacific Journal of Tropical Medicine. 2012;5(12):991-994.

12. Miranda AE, Pinto VM, McFarland W, Page K. HIV Infection Among Young Parturient Women in Brazil: prevalence and associated risk factors. AIDS Behav. 2014; 18(1):1-5.

13. Gomes DM, Oliveira MIC, Fonseca SC. Avaliação da testagem anti-HIV no pré-natal e na assistência ao parto no Rio de Janeiro, Brasil. Rev. Bras. Saúde Matern. Infant. 2015;15(4):413-423.

14. Lemos LM, Anglemyer A, Santos VS, Gurgel RQ, Rutherford GW. Prevalence of and risk factors for late diagnosis of HIV infection in Brazilian infants and children. Revista da Sociedade Brasileira de Medicina Tropical. 2015;48(3):326-30.

15. Anígilájé EA, Ageda BR, Nweke NO. Barriers to uptake of prevention of mother-to-child transmission of HIV services among mothers of vertically infected HIV-seropositive infants in Makurdi, Nigeria. Patient Prefer Adherence. 2016;10:57-72.

16. Andreatta LB, Sammarco GN. Aspecto clínico de crianças portadoras da síndrome da imunodeficiência adquirida por transmissão vertical: uma revisão bibliográfica. Revista Saúde e Pesquisa. 2012;5(2):418-423.

17. Banks LM, Zuurmond M, Ferrand R, Kuper H. The relationship between HIV and prevalence of disabilities in sub-Saharan Africa: systematic review. Trop Med Int Health. 2015;20(4):411-429.

18. Pimenta ATM, Duarteb G, Fernandezc JCC, Corread IA, Mellie PPS, Quintanaf SM. Gestantes infectadas pelo HIV-1 atendidas em serviço de referência: características clínicas e sociodemográficas. Revista de Atenção à Saúde. 2015;13(45):20-25.

Recebido em: 16/06/2018

\section{Anexos}

Tabela 1. Características dos participantes da pesquisa $(\mathrm{N}=125)$, Belém - PA, 2015

\begin{tabular}{|c|c|c|}
\hline Variáveis & $N=125$ & $\%$ \\
\hline \multicolumn{3}{|l|}{ Sexo das crianças } \\
\hline Masculino & 56 & $44,8 \%$ \\
\hline Feminino & 69 & $55,2 \%$ \\
\hline p-valor & \multicolumn{2}{|c|}{$0,28^{\mathrm{a}}$} \\
\hline \multicolumn{3}{|l|}{ Faixa etária das mães } \\
\hline 18 a 26 anos & 63 & $50,4 \%$ \\
\hline 27 anos e mais & 62 & $49,6 \%$ \\
\hline $\mathrm{p}$-valor & \multicolumn{2}{|c|}{$1,00^{\mathrm{a}}$} \\
\hline \multicolumn{3}{|l|}{ Escolaridade das mães } \\
\hline Analfabeto & 2 & $1,6 \%$ \\
\hline Ensino fundamental & 43 & $34,4 \%$ \\
\hline Ensino médio & 66 & $52,8 \%$ \\
\hline Curso superior & 5 & $4,0 \%$ \\
\hline Sem informação & 9 & $7,2 \%$ \\
\hline p-valor & \multicolumn{2}{|c|}{$<0,0001^{\mathrm{b} *}$} \\
\hline \multicolumn{3}{|l|}{ Faixa etária dos pais } \\
\hline 19 a 27 anos & 40 & $32,0 \%$ \\
\hline 28 anos e mais & 58 & $46,4 \%$ \\
\hline Não informado & 27 & $21,6 \%$ \\
\hline p-valor & \multicolumn{2}{|c|}{$0,008^{\mathrm{b} *}$} \\
\hline \multicolumn{3}{|l|}{ Escolaridade dos pais } \\
\hline Analfabeto & 4 & $3,2 \%$ \\
\hline Ensino fundamental & 36 & $28,8 \%$ \\
\hline Ensino médio & 40 & $32,0 \%$ \\
\hline Curso superior & 5 & $4,0 \%$ \\
\hline Sem informação & 40 & $32,0 \%$ \\
\hline p-valor & \multicolumn{2}{|c|}{$<0,0001^{\text {b* }}$} \\
\hline \multicolumn{3}{|l|}{ Residência } \\
\hline Rural & 43 & $34,4 \%$ \\
\hline Urbano & 82 & $65,6 \%$ \\
\hline p-valor & \multicolumn{2}{|c|}{$0,0007^{a *}$} \\
\hline
\end{tabular}

Aceito em: 20/08/2018 
Tabela 2. Exposição ao HIV dos pais das crianças participantes da pesquisa $(\mathrm{N}=125)$, Belém $(\mathrm{PA}), 2015$

\begin{tabular}{lcc}
\hline \multicolumn{1}{c}{ Variáveis } & $\begin{array}{c}\mathbf{N =} \\
\mathbf{1 2 5}\end{array}$ & $\%$ \\
\hline Exposição sexual de mãe com HIV & & \\
1 a 8 parceiros & 88 & $70,4 \%$ \\
Mais de 10 parceiros & 23 & $18,4 \%$ \\
Sem informação & 14 & $11,2 \%$ \\
p-valor & \multicolumn{2}{c}{$<0,0001^{\text {a* }}$} \\
\hline
\end{tabular}

\begin{tabular}{lcc}
\hline $\begin{array}{l}\text { Outras exposições de mães com HIV } \\
\text { (N=66) }\end{array}$ & & \\
Tatuagem e/ou piercing & 61 & $92,4 \%$ \\
Drogas inalatórias & 5 & $7,6 \%$ \\
p-valor & & $<0,0001^{\text {b* }}$ \\
\hline
\end{tabular}

Exposição sexual dos pais

\begin{tabular}{|c|c|c|}
\hline 1 a 8 parceiras & 19 & $15,2 \%$ \\
\hline Mais de 10 parceiras & 25 & $20,0 \%$ \\
\hline Sem informação & 81 & $64,8 \%$ \\
\hline p-valor & \multicolumn{2}{|c|}{$<0,0001^{\text {a* }}$} \\
\hline \multicolumn{3}{|l|}{ Bissexualidade dos pais } \\
\hline Sim & 6 & $4,8 \%$ \\
\hline Não & 119 & $95,2 \%$ \\
\hline p-valor & \multicolumn{2}{|c|}{$<0,0001^{\mathrm{b} *}$} \\
\hline \multicolumn{3}{|c|}{ Outras exposições dos pais $(\mathrm{N}=47)$} \\
\hline Tatuagem e/ou piercing & 31 & $66,0 \%$ \\
\hline Drogas inalatórias & 16 & $34,0 \%$ \\
\hline p-valor & \multicolumn{2}{|c|}{$0,04^{\mathrm{b} *}$} \\
\hline \multicolumn{3}{|c|}{ Soropositividade para o HIV nos pais } \\
\hline Positivo & 31 & $24,8 \%$ \\
\hline Negativo & 49 & $39,2 \%$ \\
\hline Não realizou o teste & 28 & $22,4 \%$ \\
\hline Sem informação & 17 & $13,6 \%$ \\
\hline p-valor & \multicolumn{2}{|c|}{$0,01^{\mathrm{a} *}$} \\
\hline
\end{tabular}

\section{Transmissão viral para criança}

\begin{tabular}{crr} 
Vertical & 125 & $100,0 \%$ \\
\hline a - Teste G (Aderência); b - Teste do Qui-quadrado; * Resultado
\end{tabular} estatisticamente significante, $\mathrm{p} \leq 0,05$.
Tabela 3. História do pré-natal de mães HIV positivas $(\mathrm{N}=125)$, Belém (PA), 2015

\begin{tabular}{lcc} 
Variáveis & $\begin{array}{c}\mathbf{N =} \\
\mathbf{1 2 5}\end{array}$ & $\%$ \\
\hline Realização do pré-natal & 120 & $96,0 \%$ \\
Sim & 5 & $4,0 \%$ \\
Não & & \\
p-valor & $<0,0001^{1 *}$ \\
\hline
\end{tabular}

Local de realização do pré-natal

UREMIA

$69 \quad 55,2 \%$

Hospitalar

$4 \quad 3,2 \%$

Particular

$4 \quad 3,2 \%$

Postos e UBS

$4132,8 \%$

Sem informação

$7 \quad 5,6 \%$

p-valor

$<0,0001^{\mathrm{b} *}$

\begin{tabular}{lcc}
\hline Número de consultas & & \\
Nenhuma & 8 & $6,4 \%$ \\
2 a 6 consultas & 51 & $48,8 \%$ \\
7 a 13 consultas & 5 & $40,8 \%$ \\
Sem informação & $<0,0001^{\mathrm{b} *}$ \\
p-valor & \multicolumn{2}{c}{} \\
\hline Descoberta da infecção por HIV & 55 & $44,0 \%$ \\
Antes da gravidez & 49 & $39,2 \%$ \\
Durante a gravidez & 17 & $13,6 \%$ \\
No parto (teste rápido) & 4 & $3,2 \%$ \\
Após o parto & $<0,0001^{\mathrm{b} *}$ \\
p-valor & \multicolumn{2}{c}{$<$} \\
\hline
\end{tabular}

\section{Terapia antirretroviral}

Sim $94 \quad 75,2 \%$

Não $\quad 31 \quad 24,8 \%$

p-valor $\quad<0,0001^{\text {a* }}$

\begin{tabular}{lcc}
\hline Terapia antirretroviral início da gestação & & \\
0 a 5 meses & 46 & $36,8 \%$ \\
6 a 9 meses & 18 & $14,4 \%$ \\
No parto & 8 & $6,4 \%$ \\
Já fazia uso do AZT & 28 & $22,4 \%$ \\
Não relata & 25 & $20,0 \%$ \\
p-valor & \multicolumn{2}{c}{$<0,0001^{\mathrm{b} *}$} \\
\hline AZT injetável & 102 & $81,6 \%$ \\
Sim & 17 & $13,6 \%$ \\
Não & \multicolumn{2}{c}{6} \\
Desconhecido & $4,8 \%$ \\
p-valor & $<0,0001^{\mathrm{b} *}$ \\
\hline
\end{tabular}

a - Teste do Qui-quadrado; b - Teste G (Aderência); * Resultado estatisticamente significante, $\mathrm{p} \leq 0,05$. 
Tabela 4. Características do parto de mães HIV positivas $(\mathrm{N}=125)$, Belém (PA), 2015

\begin{tabular}{|c|c|c|}
\hline Variáveis & $\begin{array}{c}N= \\
125\end{array}$ & $\%$ \\
\hline \multicolumn{3}{|l|}{ Local } \\
\hline Hospital público & 90 & $72,0 \%$ \\
\hline Hospitais público do interior do estado & 14 & $11,2 \%$ \\
\hline Hospitais particulares & 7 & $5,6 \%$ \\
\hline Sem informação & 14 & $11,2 \%$ \\
\hline p-valor & \multicolumn{2}{|c|}{$<0,000^{\mathrm{a}} 1^{*}$} \\
\hline \multicolumn{3}{|l|}{ Tipo de parto } \\
\hline Normal & 16 & $12,8 \%$ \\
\hline Cesárea & 109 & $87,2 \%$ \\
\hline p-valor & \multicolumn{2}{|c|}{$<0,000^{\mathrm{b}} 1^{*}$} \\
\hline \multicolumn{3}{|l|}{ Idade gestacional } \\
\hline Pré- termo & 7 & $5,6 \%$ \\
\hline A termo & 101 & $80,8 \%$ \\
\hline Pós- termo & 1 & $0,8 \%$ \\
\hline Sem informação & 16 & $12,8 \%$ \\
\hline p-valor & \multicolumn{2}{|c|}{$<0,0001^{\text {a* }}$} \\
\hline
\end{tabular}

a - Teste G (Aderência); b - Teste do Qui-quadrado; * Resultado estatisticamente significante, $\mathrm{p} \leq 0,05$

Tabela 5. Características dos recém-nascidos de mães HIV positivas $(\mathrm{N}=125)$, Belém - PA, 2015

\begin{tabular}{|c|c|c|}
\hline Variáveis & $\mathrm{N}=125$ & $\%$ \\
\hline \multicolumn{3}{|l|}{ HIV } \\
\hline Positivo & 7 & $5,6 \%$ \\
\hline Negativo & 118 & $94,4 \%$ \\
\hline p-valor & \multicolumn{2}{|c|}{$<0,0001^{\mathrm{a*}}$} \\
\hline \multicolumn{3}{|c|}{ Uso da terapia antirretroviral } \\
\hline Sim & 113 & $90,4 \%$ \\
\hline Não & 5 & $4,0 \%$ \\
\hline Desconhecido & 7 & $5,6 \%$ \\
\hline p-valor & \multicolumn{2}{|c|}{$<0,0001^{\mathrm{b} *}$} \\
\hline \multicolumn{3}{|l|}{ Aleitamento materno } \\
\hline $\operatorname{Sim}$ & 6 & $4,8 \%$ \\
\hline Não & 119 & $95,2 \%$ \\
\hline p-valor & \multicolumn{2}{|c|}{$<0,0001^{\text {a* }}$} \\
\hline \multicolumn{3}{|c|}{$\begin{array}{l}\text { Antecedentes familiares } \\
(\mathrm{N}=61)\end{array}$} \\
\hline AIDS & 23 & $37,7 \%$ \\
\hline Tuberculose & 28 & $45,9 \%$ \\
\hline Hanseníase & 10 & $16,3 \%$ \\
\hline p-valor & \multicolumn{2}{|c|}{$0,003^{\mathrm{b} *}$} \\
\hline
\end{tabular}

$\mathrm{a}$ - Teste do Qui-quadrado; b - Teste G (Aderência); * Resultado estatisticamente significante, $\mathrm{p} \leq 0,05$. 